\title{
Corticocortical Connections Mediate Primary Visual Cortex Responses to Auditory Stimulation in the Blind
}

\author{
Corinna Klinge, ${ }^{1 *}$ Falk Eippert, ${ }^{1 *}$ Brigitte Röder, ${ }^{2}$ and Christian Büchel ${ }^{1}$ \\ ${ }^{1}$ NeuroImage Nord, Department of Systems Neuroscience, University Medical Center Hamburg-Eppendorf, 20246 Hamburg, Germany, and ${ }^{2}$ Biological \\ Psychology and Neuropsychology, University of Hamburg, 20146 Hamburg, Germany
}

\begin{abstract}
Blind individuals have to rely on nonvisual information to a greater extent than sighted to efficiently interact with the environment, and consequently exhibit superior skills in their spared modalities. These performance advantages are often paralleled by responses in the occipital cortex, which have been suggested to be essential for nonvisual processing in the blind. However, it is currently unclear through which pathways (i.e., thalamocortical or corticocortical connections) nonvisual information reaches the occipital cortex of the blind. Here, we used functional magnetic resonance imaging to study blind and matched sighted humans with an auditory discrimination paradigm and used dynamic causal modeling to investigate the effective connectivity underlying auditory activations in the primary visual cortex of blind volunteers. Model comparison revealed that a model connecting the medial geniculate nucleus (MGN), primary auditory cortex (A1), and primary visual cortex (V1) in a bidirectional manner outperformed all other models in both groups. Regarding inference on model parameters, we observed that basic auditory mechanisms (i.e., sensory input to MGN and connections from MGN to A1) did not differ significantly between the two groups. In contrast, we found clear evidence for stronger corticocortical connections from A1 to V1 in the blind, whereas results with regard to thalamocortical enhancement (from MGN to V1 and, in a control analysis, from the lateral geniculate nucleus to V1) were not consistent. These results suggest that plastic changes especially in corticocortical connectivity allow auditory information to evoke responses in the primary visual cortex of blind individuals.
\end{abstract}

\section{Introduction}

Vision plays a crucial role in everyday life, making it difficult for blind people to deal with certain environmental demands. Blind individuals thus have to rely on nonvisual information to a greater extent and consequently show superior skills in their spared modalities (Röder et al., 1999b; Amedi et al., 2003; Gougoux et al., 2005). Neuroimaging studies have shown these performance enhancements to be paralleled by enhanced responses in the visual cortex [for example, audition (Weeks et al., 2000; Gougoux et al., 2009) and touch (Sadato et al., 1996; Büchel et al., 1998)] (for review, see Merabet and Pascual-Leone, 2010). Recruitment of the visual cortex in the blind has thus been hypothesized to be essential for their exceptional abilities (Hamilton and Pascual-Leone, 1998). Evidence supporting this hypothesis comes from both correlative (Gougoux et al., 2005) and causal studies (Cohen et al., 1997; Hamilton et al., 2000; Collignon et al., 2007). However, the pathways transmitting nonvisual information to visual cortex are currently unclear. Recent data shed some light on tactile input into visual cortex (Fujii et al., 2009), but the route of auditory information to the visual cortex in the blind

Received May 10, 2010; revised June 29, 2010; accepted July 29, 2010.

This work was supported by Bundesministerium für Bildung und Forschung Grant 01GW0561. We thank the radiographers of Neurolmage Nord for help with scanning and K. E. Stephan for help with analysis issues.

*C.K. and F.E. contributed equally to this work.

Correspondence should be addressed to Corinna Klinge, Department of Systems Neuroscience, University Medical Center Hamburg-Eppendorf, Martinistrasse 52, 20246 Hamburg, Germany. E-mail: c.klinge@uke. uni-hamburg.de.

DOI:10.1523/JNEUROSCI.2384-10.2010

Copyright $\odot 2010$ the authors $\quad 0270-6474 / 10 / 3012798-08 \$ 15.00 / 0$ remains to be clarified, as modality-specific recruitment mechanisms likely exist (Driver and Noesselt, 2008).

Different, possibly coexisting, routes could be mediating auditory information flow to the visual cortex in the blind (Bavelier and Neville, 2002; Driver and Noesselt, 2008): (a) reorganized thalamocortical connections or (b) corticocortical connections between primary sensory cortices [that can further be subdivided into direct long-range corticocortical connections (b1) and indirect corticocortical connections via intervening multimodal convergence zones (b2); note that, in this study, we only distinguish between thalamocortical and corticocortical connections]. Tracing studies provide structural evidence for all of these routes [(a) Karlen et al., 2006; (b1) Falchier et al., 2002; (b2) Rockland and Ojima, 2003] but do not allow to determine their functional relevance. Support for corticocortical connections comes from transcranial magnetic stimulation studies in blind humans (Wittenberg et al., 2004; Collignon et al., 2007; Ptito et al., 2008) and a resting state functional magnetic resonance imaging (fMRI) study in sighted (Eckert et al., 2008). Additional evidence for corticocortical connectivity from auditory to visual cortex comes from activation-based fMRI studies in both sighted (den Ouden et al., 2009; Werner and Noppeney, 2010) and blind humans (Fujii et al., 2009). Support for thalamocortical connections in the blind comes from a resting-state fMRI study (Liu et al., 2007), but a degeneration of the lateral geniculate nucleus (LGN) has also been observed in the blind (Noppeney et al., 2005). Hence, corticocortical connections seem more probable than thalamocortical connections to be mediating auditory responses in the visual cortex of the blind. 
Here, we analyzed fMRI data obtained during auditory tasks and investigated the effective connectivity [the influence one neural system exerts over another (Friston, 1994)] between different cortical and thalamic candidate regions via dynamic causal modeling (DCM) (Friston et al., 2003). We expected to find increased connectivity in the blind, compared with sighted, from the primary auditory cortex (A1) to the primary visual cortex (V1) but also tested for enhanced subcortical routes [via the medial geniculate nucleus (MGN) or, less likely, the LGN] to the visual cortex.

\section{Materials and Methods}

We used the data set from the study by Klinge et al. (2010), which involved auditory tasks in blind and sighted adults.

\section{Participants}

Twenty volunteers took part in the present study. Ten connatally blind (i.e., born blind) volunteers (5 males) were matched with 10 sighted participants according to gender, age, and approximate educational level (mean \pm SEM, age of blind participants, $35 \pm 3$ years; age of sighted participants, $35 \pm 3$ years) (for detailed characterization of volunteers, see Klinge et al., 2010). Participants had no neurological deficits (except for blindness) and no history of neurological or psychiatric illness, and gave written informed consent to participate. The study was approved by the local ethics committee, and participants were remunerated for participation.

\section{Stimuli, tasks, and procedure}

We used a newly developed and validated acoustic stimulus data set. Stimuli were eight different meaningless bisyllabic pseudowords (baba, babu, dede, tete, gigo, gigi, lolo, wowo), spoken by female and male professional actors in an angry, happy, neutral, or fearful voice. A total of 64 different stimuli was used in the study, with stimuli normalized to the mean sound pressure level $(80 \mathrm{~dB})$. The fMRI experiment consisted of two different tasks. In two consecutive sessions, participants either had to discriminate the emotional prosody (happy, angry, neutral, fearful) or the first vowel (a, e, i, o) of each stimulus, using individual buttons on a response pad, each corresponding to one possible answer.

Every participant went through a training session to ensure a good comprehension of the tasks, timing demands, and finger-response assignment (index and middle fingers of both hands). Trials consisted of a short acoustic warning cue $(550 \mathrm{~Hz}$ tone; $100 \mathrm{~ms})$ followed by the bisyllabic stimulus (300 ms after cue onset). Participants were asked to respond as quickly and as accurately as possible (in a time window of $2 \mathrm{~s}$ ). Average trial duration was $5.5 \mathrm{~s}$ (range, $4-7 \mathrm{~s}$; because of a jittered intertrial interval). Acoustic feedback was given in the practice session only. In the magnetic resonance (MR) scanner, all volunteers were blindfolded to guarantee for identical sensory input across groups. Stimuli were presented using MR-compatible electrodynamic headphones (MR ConFon). Volunteers were equipped with two custom-made MR-compatible response pads, one for each hand, and completed the same tasks as during training but this time without feedback. Each session consisted of 128 trials ( 16 different stimuli per emotion; each presented twice). The order of the two tasks was randomized across participants with matched volunteers receiving the same order of trials, tasks, and finger-response key assignment.

\section{Data acquisition}

fMRI data were acquired on a $3 \mathrm{~T}$ system (Trio; Siemens) equipped with a 12 channel head coil. Forty transversal slices (slice thickness, $2 \mathrm{~mm}$; $\mathrm{mm}$ gap) were acquired in each volume [repetition time (TR), $2.38 \mathrm{~s}$; echo time, $25 \mathrm{~ms}$; flip angle, $90^{\circ}$; field of view, $208 \times 208$; matrix, $104 \times$ 104; GRAPPA with PAT factor 2 and 48 reference lines], using gradient echo T2*-weighted echoplanar imaging (EPI). The first five volumes of each session and participant were discarded to control for T1 saturation effects. High-resolution $\left(1 \times 1 \times 1 \mathrm{~mm}^{3}\right.$ voxel size $)$ T1-weighted images were acquired for each participant, using an MPRAGE (magnetizationprepared rapid acquisition gradient echo) sequence.

\section{Data analysis}

fMRI data processing and statistical analyses were performed using statistical parametric mapping (SPM8; Wellcome Department of Imaging Neuroscience, London, UK). Data preprocessing consisted of slice timing (correction for differences in slice acquisition time), realignment (rigid body motion correction) and unwarping (accounting for susceptibility by movement interactions), spatial normalization to a standard EPI template (including resampling at a resolution of $2 \times 2 \times 2 \mathrm{~mm}^{3}$ ), and smoothing using a $6 \mathrm{~mm}$ (full-width at half-maximum) isotropic Gaussian kernel. Data were also subjected to high-pass filtering (cutoff period, $128 \mathrm{~s}$ ) and correction for temporal autocorrelations (based on a first-order autoregressive model). Statistical analyses were performed using a general linear model approach. On the first level, all event types (fear, happiness, neutral, and anger) were pooled and modeled as one regressor. Each event was modeled as a stick function convolved with a canonical hemodynamic response function as implemented in SPM8. Note that we pooled the two sessions into one session by concatenating the stimulus regressor, as this facilitated the DCM analyses. To compensate for possible session differences, we included two regressors that modeled each session mean; we also included one regressor that modeled the transition between the two sessions. After model estimation, the beta image reflecting the stimulus regressor from each participant was raised to the second level. At the second level, a two-sample $t$ test with nonsphericity correction (for possible unequal variance of the error term in the two groups) was performed. Group-specific contrasts and differential contrasts were computed (blind, sighted, blind $>$ sighted, and sighted $>$ blind), as well as a conjunction analysis across both groups [blind and sighted; based on the conjunction null hypothesis, testing for a logical AND (Friston et al., 2005; Nichols et al., 2005)]. We used an uncorrected threshold of $p<0.005$, but also report $p$ values for each peak that are corrected for multiple comparisons. Correction was based on cytoarchitectonic probability maps (included in the SPM anatomy toolbox as well as in FSL) (Eickhoff et al., 2005) of the following regions: MGN, A1, and V1. In a control analysis, we also included LGN. The maps of those regions were thresholded at $50 \%$ and are described in more detail in the following references [V1 (Amunts et al., 2000); A1 (Morosan et al., 2001); MGN and LGN (Bürgel et al., 2006)]. In all of the following analyses, we only focus on blood oxygen level-dependent (BOLD) responses in these regions; no whole-brain analyses were performed. For details regarding fMRI time series extraction in these regions for DCM analyses, see below.

\section{Dynamic causal modeling}

DCM is a generic approach for inferring unobserved neural states from measured brain activity. Based on a bilinear model of neural population dynamics that is combined with a hemodynamic forward model [describing the transformation of neural activity into a BOLD response (Friston et al., 2003)], three sets of parameters are estimated: (1) parameters mediating the influence of extrinsic inputs on the states (i.e., direct influence of a stimulus on regional activity), (2) parameters mediating intrinsic coupling among the states (i.e., interregional influence in the absence of experimental modulations), and (3) parameters allowing the inputs to modulate that coupling (i.e., change in the connectivity between regions induced by experimental procedures). Note that, in this paper, we are only concerned with the first two parameter sets.

Model specification. We constructed eight different models for each participant, separately for the left and right hemisphere (Fig. 1). Each model consisted of three regions: MGN, A1, and V1. The extrinsic input (auditory stimulation) always entered the system via MGN. The difference between the models consisted of the intrinsic connections that were specified between the three regions as follows: (1) a fully connected model with connections between all three regions, (2) a model with no connection between MGN and V1, (3) a model with no connection between MGN and A1, and (4) a model with no connection between A1 and V1. These four models were constructed once with bidirectional connections and once with forward connections only, forward meaning connections emanating from MGN (for a more extensive discussion of forward and backward connections in the context of DCM, see Penny et al., 2004). To allow for testing the relevance of LGN in transmitting auditory information [because of possible rewired inputs from the infe- 


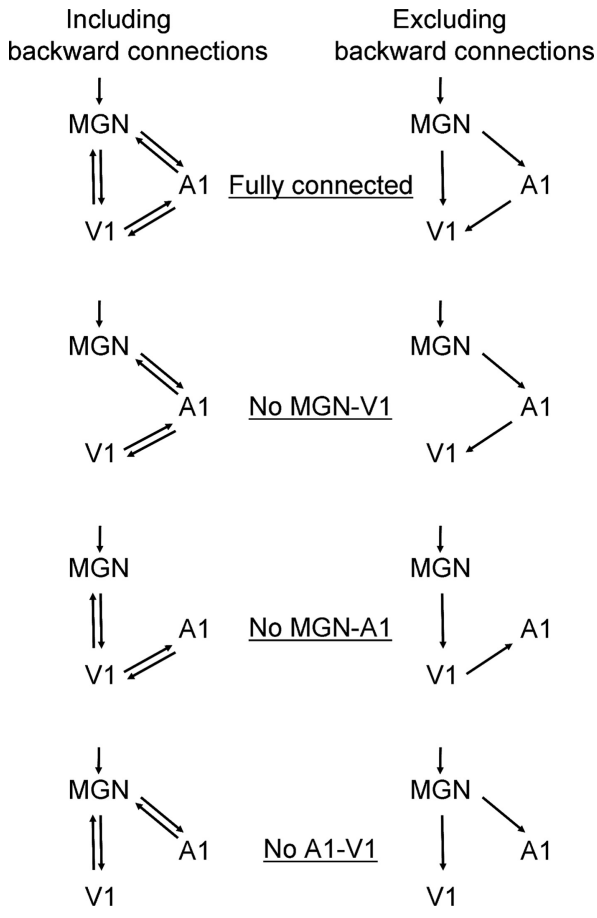

Figure 1. Schematic representation of the eight candidate dynamic causal models that were tested in this study. In all models, the driving input was on MGN, which was connected to $A 1$ and V1. The models were specified separately for each hemisphere and only differ in their intrinsic connections.

rior colliculus in the blind (Bavelier and Neville, 2002)], we also constructed models with LGN instead of MGN. Note that we did not use the extended slice-timing correction (Kiebel et al., 2007) during model specification, but instead used SPMs original slice-timing correction, because we had a short TR $(2.38 \mathrm{~s})$ and our regions of interest had a maximal slice difference of six slices. In such situations, the original slice-timing correction performs well (Kiebel et al., 2007).

Time series extraction. DCM rests on fMRI time series extracted from activation peaks in different regions, which comprised MGN, A1, and V1 in our study. We took special care in selecting only time series from coordinates that were clearly located within the primary sensory cortices and the MGN, by using cytoarchitectonic maps of these three regions to constrain the search volume (see also below). An additional important point in DCM analyses with regard to group differences is to use coordinates for time series extraction that do not bias the later DCM analysis. For example, using coordinates for time series extraction from a location at which only one group shows significant activations is likely to bias the estimates of effective connectivity to and from that region. We therefore used coordinates obtained from the random effects conjunction analysis as a starting point for time series extraction. This ensured not only that similar anatomical loci would be the basis of the DCM analysis in both groups but also that significant responses would be present in both groups at the identified location.

Because local maxima of activation differ across individuals, we did not use the coordinates of group activation peaks for fMRI time series extraction, but instead used individually adjusted coordinates for each participant, by using a combination of functional and anatomical constraints in each of the three regions (MGN, A1, and V1). In each region of each participant, we determined the coordinates for fMRI time series extraction by identifying the most significant activation peak that satisfied three conditions: (1) it had to be significant at a level of $p<0.05$ uncorrected, (2) it had to be within a sphere around the group peak of the conjunction analysis ( $4 \mathrm{~mm}$ radius for MGN [and LGN]; $8 \mathrm{~mm}$ radius for $\mathrm{A} 1$ and V1), and (3) it had to be within the cytoarchitectonically defined mask. After identifying an individual peak, we placed a sphere around this peak [ $4 \mathrm{~mm}$ radius for MGN (and LGN); $8 \mathrm{~mm}$ radius for $\mathrm{Al}$ and $\mathrm{V} 1$ ] and extracted the first eigenvector of the time series; adjustment was also performed to remove unspecific effects (session mean and session transitions).

If a participant did not show significant BOLD responses in a search region (i.e., the intersection of sphere and mask), we instead used the group coordinates of activation in that region for time series extraction, because we had a rather small sample size (10 blind and 10 sighted adults). Supplemental Table 1 (available at www.jneurosci.org as supplemental material) lists the coordinates of each peak from each participant and also indicates whether a participant did not show significant responses in a search region. Substitution with group coordinates was necessary in only 11 (4 blind, 7 sighted) of 120 cases (i.e., 20 subjects with 3 regions in each hemisphere). Note that we controlled for this potential confound and also performed an analysis in which only participants with significant responses were included.

In two additional control analyses (for more details, see Results), we did not use the conjunction analysis to identify second-level group peaks, but instead used the simple within-group contrasts.

Inference on model space (Bayesian model selection). To identify the most likely model, we used random-effects Bayesian model selection (BMS) as implemented in SPM8 (Stephan et al., 2009). This procedure enables one to identify - among the whole set of models - the model that best fits the data but at the same time is least complex (i.e., ideal balance between accuracy and complexity). Balancing model fit with model complexity is necessary to avoid overfitting and to allow generalization (Pitt and Myung, 2002). The random-effects BMS technique is robust against outliers and more sensitive than classical frequentist approaches if high group heterogeneity is present (Stephan et al., 2009).

The results of the inference on model space using BMS are reported using the exceedance probability $\varphi_{k}$, which is the probability that a specific model $k$ is more likely than any of the other models contained in model space. Note that the exceedance probability $\varphi$ sums to 1 over all models included in the BMS procedure. BMS was used for each hemisphere and group separately to identify the optimal model. Model space consisted of the eight previously specified models (see above, Model specification). We were only interested in identifying the single optimal model in each group and hemisphere, although our model construction approach would in principle allow two-factorial model space partitioning and subsequent model-family comparison (Stephan et al., 2009).

Some authors have also performed model comparison on a different part of model space, namely on the location at which the extrinsic input enters the system (Ethofer et al., 2006; Vaudano et al., 2009). However, such a model comparison is not necessary in our study, as it is unequivocally established that the MGN is the major auditory input to A1 (Hudspeth, 2000).

Inference on model parameters. After identification of the optimal model in each group and hemisphere via random-effects Bayesian model selection, we proceeded to test the parameter estimates of both input and intrinsic connections of the winning model for significance using a random-effects approach (Stephan et al., 2010). Regarding group differences, we only had hypotheses for the MGN-V1 and A1-V1 connections, but also tested the MGN-A1 connection and the input to MGN for possible group differences using two-sample $t$ tests. We used the Bonferroni correction to adjust the $p$ value for the number of tests within each parameter class (i.e., three tests, $p<0.017$ for intrinsic connections), although this correction for multiple comparisons is rather conservative in the presence of dependencies among the parameters. As we expected stronger connections from $\mathrm{A} 1$ to $\mathrm{V} 1$ and possibly from MGN to $\mathrm{V} 1$ in the blind, we used one-tailed two-sample $t$ tests in these cases, but two-tailed two-sample $t$ tests otherwise. We also report within-group results of input to MGN and all intrinsic connections using a one-sample $t$ test (two-tailed).

\section{Results}

First, we investigated whether BOLD responses would differ between the groups in our regions of interest (MGN, A1, and V1). When testing for greater BOLD responses in the blind than in the sighted, we observed a clear enhancement of BOLD responses in 
Table 1. Results of the conjunction analysis across both groups (blind and sighted)

\begin{tabular}{|c|c|c|c|c|c|c|c|c|}
\hline & \multicolumn{4}{|l|}{ Left hemisphere } & \multicolumn{4}{|c|}{ Right hemisphere } \\
\hline & Coordinates & $T_{(18)}$ & $p$ (uncorrected) & $p$ (corrected) & Coordinates & $T_{(18)}$ & $p$ (uncorrected) & $p$ (corrected) \\
\hline MGN & $-14-26-6$ & 3.11 & 0.003 & 0.014 & $16-24-6$ & 3.9 & 0.001 & 0.004 \\
\hline $\mathrm{A} 1$ & $-54-142$ & 9.73 & 0.000 & 0.000 & $54-146$ & 9.71 & 0.000 & 0.000 \\
\hline V1 & $-6-746$ & 3.9 & 0.001 & 0.160 & $4-808$ & 3.7 & 0.001 & 0.275 \\
\hline
\end{tabular}

Coordinates are denoted in millimeters (Montreal Neurological Institute space). Corrected $p$ values refer to the respective search volume (based on cytoarchitectonic probability maps).
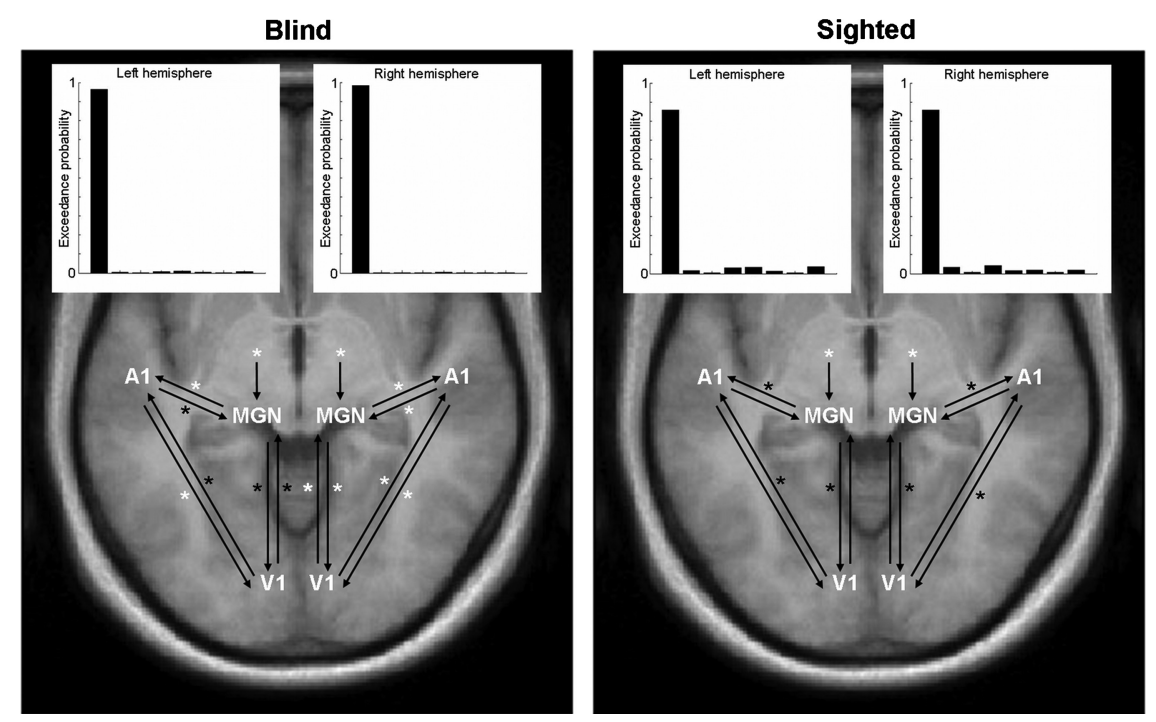

Figure 2. Model comparison results and parameter estimates of inputs and intrinsic connections for each group. The white insets show the results of the random-effects Bayesian model comparison procedure, with bars indicating the exceedance probability for each of the eight models shown in Figure 1. The winning model in both hemispheres and groups was the fully connected model (first bar). The within-group strength of the input and intrinsic connections of the winning model are represented by the asterisks in the bottom part of the figure (black, $p<0.05$, uncorrected; white, $p<0.05$, corrected; two-tailed testing). The exact scores can be found in Table 2. The background image is a transversal slice of the average structural image of all participants (at $z=-6 \mathrm{~mm}$, the location of the MGN peaks).

bilateral V1 (supplemental Table 2, supplemental Fig. 1, available at www.jneurosci.org as supplemental material), but not in MGN or A1. The sighted did not show significantly greater responses than the blind in MGN or V1, but showed slightly enhanced responses within bilateral A1 (supplemental Table 2, available at www. jneurosci.org as supplemental material). To avoid any bias for subsequent DCM analyses, we used a conjunction analysis over both groups to obtain coordinates for the DCM analyses. This ensured that (1) both groups showed significant responses at the identified peak and (2) time courses from identical regions entered the DCM analysis. Across both groups, significant BOLD responses were observed in all three regions in both hemispheres (Table 1; supplemental Fig. 2, available at www.jneurosci.org as supplemental material). Importantly, these responses did not differ significantly between the two groups at the identified voxels (not even at a liberal threshold of $p<0.05$, uncorrected).

Random-effects BMS was then used within each group and hemisphere to determine the optimal model in explaining the data. As can be seen in Figure 2, the fully connected model clearly outperformed all other models in both hemispheres (exceedance probability of $\varphi>0.85$ in all cases). Importantly, this effect was evident in both groups, suggesting that this low-level neuroarchitecture is similar in both blind and sighted adults. Having identified the winning model, we tested the within-group input and connection strengths for significance (Fig. 2; Table 2). Not surprisingly, the auditory driving inputs on MGN were highly significant in both hemispheres of each group. Connections from
MGN to A1 were positive and by far the strongest. Backward connections were generally weaker than forward connections. The only nonsignificant connections were found in the sighted group, in which several backward connections and the connection from left A1 to left V1 failed to reach significance.

Regarding group differences in connection strengths, A1-V1 connections were significantly stronger in the blind than in the sighted (Fig. 3), both in the left hemisphere $\left(t_{(18)}=2.48 ; p=0.012\right)$ and in the right hemisphere $\left(t_{(18)}=3.23 ; p=\right.$ $0.002)$; both effects survived correction for multiple comparisons. MGN-V1 connections tended to be greater in the blind than in the sighted in the right hemisphere $\left(t_{(18)}=1.16 ; p=0.057\right)$ but were far from statistical significance when considering the need to correct for multiple comparisons; the left hemisphere showed no group difference $\left(t_{(18)}=-0.35\right.$; NS) (supplemental Fig. $3 a$, available at www. jneurosci.org as supplemental material). No significant group differences were found for the MGN-A1 connection and the input to MGN (all $p>0.2$ ). Note that the effect of enhanced A1-V1 connectivity remained stable (although to a slightly lesser extent), when including only participants who showed significant BOLD responses within the search region in each region of interest in the first-level analysis (supplemental Table 3, supplemental Fig. 4, available at www.jneurosci. org as supplemental material).

In an additional analysis, we substituted MGN with LGN to test for the possibility that LGN is differentially recruited in the blind to relay auditory information to visual cortex. LGN BOLD responses (as obtained via the conjunction analysis) were rather weak and survived neither the uncorrected threshold $(p<0.005)$ nor the correction for multiple comparisons [left LGN: $-22-24$ $-6, T_{(18)}=2.00, p=0.03$ (uncorrected), $p=0.13$ (corrected); right LGN: $24-24-6, T_{(18)}=2.45, p=0.01$ (uncorrected), $p=0.07$ (corrected)]. LGN connection strength to V1 was not enhanced in the blind compared with the sighted but nonsignificantly reduced (supplemental Fig. 3b, available at www.jneurosci.org as supplemental material).

The main finding of enhanced connections from A1 to V1 in the blind was obtained using BOLD time courses from a region of $\mathrm{V} 1$ that showed significant responses in both groups as identified via a conjunction analysis. This approach-which has also been taken by other investigators looking at group differences in connectivity (Fujii et al., 2009) — ensures that fMRI time series from similar locations in both groups enter the DCM analysis. However, these regions are not necessarily the ones in which maximal 
Table 2. Parameter estimates of inputs and intrinsic connections for each group and hemisphere

\begin{tabular}{|c|c|c|c|c|c|c|c|c|}
\hline \multirow[b]{3}{*}{ Parameter } & \multicolumn{4}{|l|}{ Left hemisphere } & \multicolumn{4}{|l|}{ Right hemisphere } \\
\hline & \multicolumn{2}{|l|}{ Blind } & \multicolumn{2}{|l|}{ Sighted } & \multicolumn{2}{|l|}{ Blind } & \multicolumn{2}{|l|}{ Sighted } \\
\hline & Mean \pm SEM & $p$ & Mean \pm SEM & $p$ & Mean \pm SEM & $p$ & Mean \pm SEM & $p$ \\
\hline Input MGN & $1.05 \pm 0.24$ & 0.002 & $1.16 \pm 0.24$ & 0.001 & $1.16 \pm 0.11$ & 0.000 & $1.03 \pm 0.29$ & 0.006 \\
\hline MGN-A1 & $0.63 \pm 0.16$ & 0.004 & $0.55 \pm 0.20$ & 0.024 & $0.77 \pm 0.06$ & 0.000 & $0.50 \pm 0.21$ & 0.042 \\
\hline A1-MGN & $-0.10 \pm 0.03$ & 0.010 & $-0.16 \pm 0.09$ & 0.087 & $-0.15 \pm 0.02$ & 0.000 & $-0.17 \pm 0.10$ & 0.143 \\
\hline MGN-V1 & $0.24 \pm 0.11$ & 0.047 & $0.29 \pm 0.11$ & 0.022 & $0.40 \pm 0.04$ & 0.000 & $0.23 \pm 0.09$ & 0.030 \\
\hline V1-MGN & $-0.10 \pm 0.04$ & 0.038 & $-0.01 \pm 0.02$ & 0.797 & $-0.17 \pm 0.02$ & 0.000 & $-0.05 \pm 0.02$ & 0.058 \\
\hline $\mathrm{A} 1-\mathrm{V} 1$ & $0.25 \pm 0.07$ & 0.004 & $0.05 \pm 0.05$ & 0.364 & $0.29 \pm 0.05$ & 0.000 & $0.10 \pm 0.04$ & 0.021 \\
\hline V1-A1 & $0.15 \pm 0.05$ & 0.020 & $0.24 \pm 0.10$ & 0.046 & $0.16 \pm 0.05$ & 0.007 & $0.18 \pm 0.09$ & 0.074 \\
\hline
\end{tabular}

The $p$ values refer to the within-group test (two-tailed one-sample $t$ test). To survive correction for multiple comparisons (Bonferroni), the $p$ value has to fall below 0.009 .
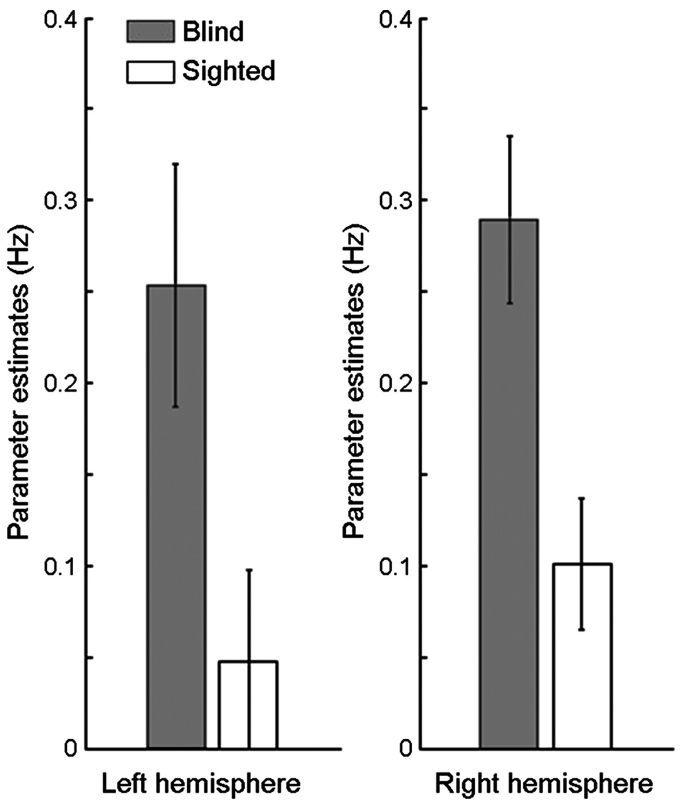

Figure 3. Connection from A1 to V1. In both hemispheres, the connection from A1 to V1 was significantly stronger in the blind group. This effect survived correction for multiple comparisons and was replicated in several control analyses. Error bars indicate SEM.

BOLD responses are observed in each group. We therefore performed a complimentary control analysis, in which we substituted the V1 peak as identified via the conjunction analysis with group-specific V1 peaks [blind group left hemisphere; $-2-884$, $T_{(18)}=7.48, p<0.001$ (uncorrected), $p=0.001$ (corrected); blind group right hemisphere: $20-10010, T_{(18)}=8.89, p<$ 0.001 (uncorrected), $p<0.001$ (corrected); sighted group left hemisphere: $-6-746, T_{(18)}=3.90, p=0.001$ (uncorrected), $p=0.160$ (corrected); sighted group right hemisphere: $10-66$ $14, T_{(18)}=3.76, p=0.001$ (uncorrected), $p=0.254$ (corrected)]. BMS again favored the fully connected model in both hemispheres of each group (exceedance probability of $\varphi>0.85$ in all cases). Similarly, we again observed a significant enhancement of the connection from $\mathrm{A} 1$ to $\mathrm{V} 1$ in both hemispheres in the blind (left: $T_{(18)}=2.58, p=0.010$; right: $T_{(18)}=2.96, p=0.004$ ), but no significant difference in the path from MGN to V1 (left: $T_{(18)}=$ -0.40 , NS; right: $T_{(18)}=0.21$, NS); no group differences were observed for the MGN-A1 connection and the input on MGN (all $p>0.2$ ). In an additional control analysis, we used all voxels in V1 that were significant in each participant's first-level analysis (at a liberal threshold of $p<0.05$ uncorrected; note that every participant showed significant responses in bilateral V1). Although this analysis does not have any localizing power within
V1, it represents a summary measure of each participant's BOLD responses within V1. Consistent with the above-mentioned greater BOLD responses in the blind, we also observed that the number of voxels that were significant in each participant (i.e., the spatial extent of activation) was significantly higher in the blind than in the sighted in both hemispheres (left: $T_{(18)}=4.34$, $p<0.001$; right: $\left.T_{(18)}=3.32, p=0.002\right)$. BMS clearly favored the fully connected model (exceedance probability of $\varphi>0.87$ in all cases). The corticocortical connection from A1 to V1 was again enhanced in the blind in both hemispheres (left: $T_{(18)}=2.09, p=$ 0.026; right: $\left.T_{(18)}=2.91, p=0.005\right)$, although the effect was slightly smaller than in the other analyses and did not survive correction for multiple comparisons in the left hemisphere. No significant differences were observed in the connection from MGN to V1 (left: $T_{(18)}=-0.21$, NS; right: $T_{(18)}=0.52, \mathrm{NS}$ ).

\section{Discussion}

In this study, we investigated possible pathways of auditory information transfer to the primary visual cortex of blind human adults and sighted controls, using dynamic causal modeling in the context of an auditory discrimination fMRI paradigm. We identified a fully connected model (bidirectional connections between MGN, A1, and V1) as the best model in both groups. Focusing on both corticocortical and thalamocortical connections, we observed clear evidence for enhanced corticocortical connections from A1 to $\mathrm{V} 1$ in the blind compared with the sighted in both hemispheres, whereas thalamocortical connections did not show consistent effects.

The auditory discrimination task we used led to significant activation in bilateral medial geniculate nucleus, primary auditory cortex, and primary visual cortex in both groups. The activation of primary visual cortex in response to auditory stimulation in the sighted might seem surprising at first sight, but note that previous studies have shown nonvisual responses in primary visual cortex of sighted participants (Maeder et al., 2001; Saito et al., 2005; Cate et al., 2009). More generally, both monkey and human studies have shown that traditional views of unimodal primary cortices have to be reconsidered, as primary cortices also respond to stimuli from other modalities, although to a lesser degree (for review, see Driver and Noesselt, 2008). We also observed that sighted participants showed slightly stronger responses in primary auditory cortex than the blind, a phenomenon that has been observed previously (Gougoux et al., 2009) and has been hypothesized to result from a more widespread auditory network toward visual areas in the blind (but see Röder et al., 1999a). Most importantly, we observed clearly enhanced responses in the primary visual cortex of the blind compared with the sighted, as has been shown before, using, for example, auditory (Weeks et al., 2000; Röder et al., 2002; Gougoux et al., 2009) or tactile stimuli (Sadato et al., 
1996; Röder et al., 1997; Büchel et al., 1998) (for review, see Merabet and Pascual-Leone, 2010). Having identified these enhanced V1 BOLD responses to auditory stimulation, we used dynamic causal modeling to test possible pathways mediating this effect.

According to previous ideas that were based on anatomical and functional studies in both animals and humans (for review, see Bavelier and Neville, 2002; Driver and Noesselt, 2008), auditory information can reach the primary visual cortex both via thalamocortical and corticocortical connections (either connecting A1 to V1 directly or via multisensory regions). Our modeling results suggest enhanced corticocortical connectivity as opposed to thalamocortical connectivity in blind relative to sighted subjects. We took great care in standardizing the selection of time series across subjects and groups: the choice of subject-specific regional time series was constrained both functionally (using a conjunction analysis across groups) and anatomically (by probabilistic cytoarchitectonic atlases for objective definition of anatomical regions). Furthermore, the effect was very robust and also held true when performing several control analyses, such as using group-specific activation peaks or using a summary measure of individual V1 BOLD responses. We did not find consistent evidence for enhanced thalamocortical connections in the blind. Only in one of the analyses did we observe a trend for enhanced connectivity from MGN to V1 in the right hemisphere, but this effect did not survive correction for multiple comparisons and was not evident in our control analyses. Although human imaging studies have repeatedly indicated a degeneration of LGN (Noppeney et al., 2005; Shimony et al., 2006) (but see Bridge et al., 2009), we also tested for enhanced LGN-V1 connectivity in the blind to investigate whether a rewiring could have taken place in the blind and to exclude unspecific thalamic spillover effects as a possible explanation. We did not observe an enhancement in the blind, but rather a slight (nonsignificant) reduction in LGN-V1 coupling compared with the sighted, which fits with the observed degeneration results. As statistical comparisons between connection strengths from different models are not valid (Stephan et al., 2010), we only note descriptively that MGN-V1 coupling seems to be stronger in the blind than LGN-V1 coupling, indicating that auditory LGN contributions to visual cortex BOLD responses via rewired input from the inferior colliculus are unlikely. We did not include nonspecific (multisensory) thalamic nuclei, such as the interlaminar nuclei (Berman, 1991) in our analyses, as such multiple differentiation of thalamic regions would necessitate additional imaging modalities (e.g., diffusion tensor imaging). Together, our data suggest that—at least for audition-predominantly corticocortical connections seem to mediate the enhanced BOLD responses in the primary visual cortex of blind participants.

As we performed an fMRI study, we can only speculate about the physiological mechanisms underlying this form of plastic change in corticocortical connectivity. Developmental changes in local connectivity [whether they may be pruning of exuberant connections, masking of silent synapses, or active inhibition (Bavelier and Neville, 2002; Maurer et al., 2005)] likely differ between healthy and visually deprived individuals. It has been suggested that unmasking of preexisting connections and shifts in connectivity might underlie rapid, early plastic changes, as for example, after a couple of days of blindfolding (Merabet et al., 2008). If sustained and reinforced, these can then lead to slower but more permanent structural changes, such as dendritic arborization, sprouting, and growth with rewiring of connections (Pascual-Leone et al., 2005). The earlier the sensory loss, the more striking the neuroplastic effects (Hensch, 2005). One could thus say that it is the input that determines which connection gets pruned and which is left unchanged (Sur et al., 1988; Sharma et al., 2000; von Melchner et al., 2000). If a person lacks visual input, the natural pruning process could be disturbed, leaving exuberant connections, as also indicated by recent findings of increased cortical thickness in the visual cortex of blind volunteers (Jiang et al., 2009).

Although we observed a clearly enhanced pathway from A1 to $\mathrm{V} 1$ in connatally blind adults, this measure of effective connectivity does not allow us to differentiate between a direct (monosynaptic) pathway linking the two primary cortices and an indirect (polysynaptic) pathway via multisensory convergence zones. To do so, one would have to include candidate regions that mediate audiovisual integration and are connected to both sensory cortices in the models [such as the superior temporal sulcus, but also parietal, premotor, and prefrontal regions (Driver and Noesselt, 2008)]. Furthermore, evidence from primates indicates that direct long-range connections also exist between nonprimary sensory areas (Rockland and Ojima, 2003). We decided against testing for all these possibilities, as on the one hand model space would become extremely large, and on the other hand cytoarchitectonic information is currently not available for these areas, thus limiting objective identification. In a recent study on tactile processing in blind participants, Fujii et al. (2009) included more regions in their DCM analyses, potentially enabling them to differentiate between direct or feedback connections, which led them to favor a model comprising indirect connections between primary somatosensory cortex and primary visual cortex via parietal regions. It is important to keep in mind, however, that there likely are differences between modalities regarding effective connectivity between the regions involved (Driver and Noesselt, 2008). In line with this argument, in a very recent study of audiovisual integration, Werner and Noppeney (2010) reported evidence for both direct corticocortical and indirect corticocortical pathways in humans during audiovisual object categorization.

A finding that initially seems at odds with our data is that Liu et al. (2007) and Yu et al. (2008) reported decreased functional connectivity [i.e., correlations of the BOLD signal between brain regions (Friston et al., 1993)] in blind volunteers compared with controls between visual and auditory cortical areas during resting-state fMRI. However, if one considers that the visual cortex can also be activated by auditory stimuli in sighted if the task requires high levels of attention (Cate et al., 2009), the results seem more alike: possibly, the visual cortex of blind people is predominantly recruited during demanding tasks or when relevant information is supplied (Röder et al., 1996). This would explain why resting-state studies (no attentional demands) failed to find coupling (Liu et al., 2007; Yu et al., 2008), whereas activation-based effective-connectivity studies did find enhanced coupling in the blind (Fujii et al., 2009; present study). This hypothesis is also backed by studies that found attentional modulations of visual cortex activity in the blind (Liotti et al., 1998; Stevens et al., 2007).

In conclusion, we found that enhanced BOLD responses to auditory stimuli in the primary visual cortex of blind volunteers are mediated by corticocortical connections from the primary auditory cortex rather than by thalamocortical connections. It will be interesting to see whether the pattern we observed is of a more general nature (i.e., also underlies multisensory integration and will for instance hold when presenting deaf people with visual stimuli [leading to activation of auditory cortex (Nishimura et al., 1999; Finney et al., 2001)]), which should result in en- 
hanced connections from V1 to A1. Whatever the case, our results clearly indicate that, during an auditory task, the deprived visual cortex of blind participants is recruited by cortical connections emanating from the primary auditory cortex.

\section{References}

Amedi A, Raz N, Pianka P, Malach R, Zohary E (2003) Early "visual" cortex activation correlates with superior verbal memory performance in the blind. Nat Neurosci 6:758-766.

Amunts K, Malikovic A, Mohlberg H, Schormann T, Zilles K (2000) Brodmann's areas 17 and 18 brought into stereotaxic space-where and how variable? Neuroimage 11:66-84.

Bavelier D, Neville HJ (2002) Cross-modal plasticity: where and how? Nat Rev Neurosci 3:443-452.

Berman NE (1991) Alterations of visual cortical connections in cats following early removal of retinal input. Brain Res Dev Brain Res 63:163-180.

Bridge H, Cowey A, Ragge N, Watkins K (2009) Imaging studies in congenital anophthalmia reveal preservation of brain architecture in "visual" cortex. Brain 132:3467-3480.

Büchel C, Price C, Frackowiak RS, Friston K (1998) Different activation patterns in the visual cortex of late and congenitally blind subjects. Brain 121:409-419.

Bürgel U, Amunts K, Hoemke L, Mohlberg H, Gilsbach JM, Zilles K (2006) White matter fiber tracts of the human brain: three-dimensional mapping at microscopic resolution, topography and intersubject variability. Neuroimage 29:1092-1105.

Cate AD, Herron TJ, Yund EW, Stecker GC, Rinne T, Kang X, Petkov CI, Disbrow EA, Woods DL (2009) Auditory attention activates peripheral visual cortex. PLoS One 4:e4645.

Cohen LG, Celnik P, Pascual-Leone A, Corwell B, Falz L, Dambrosia J, Honda M, Sadato N, Gerloff C, Catalá MD, Hallett M (1997) Functional relevance of cross-modal plasticity in blind humans. Nature 389:180-183.

Collignon O, Lassonde M, Lepore F, Bastien D, Veraart C (2007) Functional cerebral reorganization for auditory spatial processing and auditory substitution of vision in early blind subjects. Cereb Cortex 17:457-465.

den Ouden HE, Friston KJ, Daw ND, McIntosh AR, Stephan KE (2009) A dual role for prediction error in associative learning. Cereb Cortex 19:1175-1185.

Driver J, Noesselt T (2008) Multisensory interplay reveals crossmodal influences on "sensory-specific" brain regions, neural responses, and judgments. Neuron 57:11-23.

Eckert M, Kamdar N, Chang C, Beckmann C, Greicius M, Menon V (2008) A cross-modal system linking primary auditory and visual cortices: evidence from intrinsic fMRI connectivity analysis. Hum Brain Mapp 29: $848-857$.

Eickhoff SB, Stephan KE, Mohlberg H, Grefkes C, Fink GR, Amunts K, Zilles K (2005) A new SPM toolbox for combining probabilistic cytoarchitectonic maps and functional imaging data. Neuroimage 25:1325-1335.

Ethofer T, Anders S, Erb M, Herbert C, Wiethoff S, Kissler J, Grodd W, Wildgruber D (2006) Cerebral pathways in processing of affective prosody: a dynamic causal modeling study. Neuroimage 30:580-587.

Falchier A, Clavagnier S, Barone P, Kennedy H (2002) Anatomical evidence of multimodal integration in primate striate cortex. J Neurosci 22:5749-5759.

Finney EM, Fine I, Dobkins KR (2001) Visual stimuli activate auditory cortex in the deaf. Nat Neurosci 4:1171-1173.

Friston KJ (1994) Functional and effective connectivity in neuroimaging: a synthesis. Hum Brain Mapp 2:56-78.

Friston KJ, Frith CD, Liddle PF, Frackowiak RS (1993) Functional connectivity: the principal-component analysis of large (PET) data sets. J Cereb Blood Flow Metab 13:5-14.

Friston KJ, Harrison L, Penny W (2003) Dynamic causal modelling. Neuroimage 19:1273-1302.

Friston KJ, Penny WD, Glaser DE (2005) Conjunction revisited. Neuroimage 25:661-667.

Fujii T, Tanabe HC, Kochiyama T, Sadato N (2009) An investigation of cross-modal plasticity of effective connectivity in the blind by dynamic causal modeling of functional MRI data. Neurosci Res 65:175-186.

Gougoux F, Zatorre RJ, Lassonde M, Voss P, Lepore F (2005) A functional neuroimaging study of sound localization: visual cortex activity predicts performance in early-blind individuals. PLoS Biol 3:e27.

Gougoux F, Belin P, Voss P, Lepore F, Lassonde M, Zatorre RJ (2009) Voice perception in blind persons: a functional magnetic resonance imaging study. Neuropsychologia 47:2967-2974.

Hamilton R, Pascual-Leone A (1998) Cortical plasticity associated with Braille learning. Trends Cogn Sci 2:168-174.

Hamilton R, Keenan JP, Catala M, Pascual-Leone A (2000) Alexia for Braille following bilateral occipital stroke in an early blind woman. Neuroreport 11:237-240.

Hensch TK (2005) Critical period plasticity in local cortical circuits. Nat Rev Neurosci 6:877-888.

Hudspeth AJ (2000) Hearing. In: Principles of neural science (Kandel ER, Schwartz JH, Jessell TM, eds), pp 590-613. New York: McGraw-Hill.

Jiang J, Zhu W, Shi F, Liu Y, Li J, Qin W, Li K, Yu C, Jiang T (2009) Thick visual cortex in the early blind. J Neurosci 29:2205-2211.

Karlen SJ, Kahn DM, Krubitzer L (2006) Early blindness results in abnormal corticocortical and thalamocortical connections. Neuroscience 142:843858.

Kiebel SJ, Klöppel S, Weiskopf N, Friston KJ (2007) Dynamic causal modeling: a generative model of slice timing in fMRI. Neuroimage 34:1487-1496.

Klinge C, Röder B, Büchel C (2010) Increased amygdala activation to emotional auditory stimuli in the blind. Brain 133:1729-1736.

Liotti M, Ryder K, Woldorff MG (1998) Auditory attention in the congenitally blind: where, when and what gets reorganized? Neuroreport 9:1007-1012.

Liu Y, Yu C, Liang M, Li J, Tian L, Zhou Y, Qin W, Li K, Jiang T (2007) Whole brain functional connectivity in the early blind. Brain 130:2085-2096.

Maeder PP, Meuli RA, Adriani M, Bellmann A, Fornari E, Thiran JP, Pittet A, Clarke S (2001) Distinct pathways involved in sound recognition and localization: a human fMRI study. Neuroimage 14:802-816.

Maurer D, Lewis TL, Mondloch CJ (2005) Missing sights: consequences for visual cognitive development. Trends Cogn Sci 9:144-151.

Merabet LB, Pascual-Leone A (2010) Neural reorganization following sensory loss: the opportunity of change. Nat Rev Neurosci 11:44-52.

Merabet LB, Hamilton R, Schlaug G, Swisher JD, Kiriakopoulos ET, Pitskel NB, Kauffman T, Pascual-Leone A (2008) Rapid and reversible recruitment of early visual cortex for touch. PLoS One 3:e3046.

Morosan P, Rademacher J, Schleicher A, Amunts K, Schormann T, Zilles K (2001) Human primary auditory cortex: cytoarchitectonic subdivisions and mapping into a spatial reference system. Neuroimage 13:684-701.

Nichols T, Brett M, Andersson J, Wager T, Poline JB (2005) Valid conjunction inference with the minimum statistic. Neuroimage 25:653-660.

Nishimura H, Hashikawa K, Doi K, Iwaki T, Watanabe Y, Kusuoka H, Nishimura T, Kubo T (1999) Sign language "heard" in the auditory cortex. Nature 397:116.

Noppeney U, Friston KJ, Ashburner J, Frackowiak R, Price CJ (2005) Early visual deprivation induces structural plasticity in gray and white matter. Curr Biol 15:R488-R490.

Pascual-Leone A, Amedi A, Fregni F, Merabet LB (2005) The plastic human brain cortex. Annu Rev Neurosci 28:377-401.

Penny WD, Stephan KE, Mechelli A, Friston KJ (2004) Comparing dynamic causal models. Neuroimage 22:1157-1172.

Pitt MA, Myung IJ (2002) When a good fit can be bad. Trends Cogn Sci 6:421-425.

Ptito M, Fumal A, de Noordhout AM, Schoenen J, Gjedde A, Kupers R (2008) TMS of the occipital cortex induces tactile sensations in the fingers of blind Braille readers. Exp Brain Res 184:193-200.

Rockland KS, Ojima H (2003) Multisensory convergence in calcarine visual areas in macaque monkey. Int J Psychophysiol 50:19-26.

Röder B, Rösler F, Hennighausen E, Näcker F (1996) Event-related potentials during auditory and somatosensory discrimination in sighted and blind human subjects. Brain Res Cogn Brain Res 4:77-93.

Röder B, Rösler F, Hennighausen E (1997) Different cortical activation patterns in blind and sighted humans during encoding and transformation of haptic images. Psychophysiology 34:292-307.

Röder B, Rösler F, Neville HJ (1999a) Effects of interstimulus interval on auditory event-related potentials in congenitally blind and normally sighted humans. Neurosci Lett 264:53-56.

Röder B, Teder-Sälejärvi W, Sterr A, Rösler F, Hillyard SA, Neville HJ (1999b) Improved auditory spatial tuning in blind humans. Nature 400:162-166.

Röder B, Stock O, Bien S, Neville H, Rösler F (2002) Speech processing 
activates visual cortex in congenitally blind humans. Eur J Neurosci 16:930-936.

Sadato N, Pascual-Leone A, Grafman J, Ibañez V, Deiber MP, Dold G, Hallett M (1996) Activation of the primary visual cortex by Braille reading in blind subjects. Nature 380:526-528.

Saito DN, Yoshimura K, Kochiyama T, Okada T, Honda M, Sadato N (2005) Cross-modal binding and activated attentional networks during audiovisual speech integration: a functional MRI study. Cereb Cortex 15:1750-1760.

Sharma J, Angelucci A, Sur M (2000) Induction of visual orientation modules in auditory cortex. Nature 404:841-847.

Shimony JS, Burton H, Epstein AA, McLaren DG, Sun SW, Snyder AZ (2006) Diffusion tensor imaging reveals white matter reorganization in early blind humans. Cereb Cortex 16:1653-1661.

Stephan KE, Penny WD, Daunizeau J, Moran RJ, Friston KJ (2009) Bayesian model selection for group studies. Neuroimage 46:1004-1017.

Stephan KE, Penny WD, Moran RJ, den Ouden HE, Daunizeau J, Friston KJ (2010) Ten simple rules for dynamic causal modeling. Neuroimage 49:3099-3109.

Stevens AA, Snodgrass M, Schwartz D, Weaver K (2007) Preparatory activity in occipital cortex in early blind humans predicts auditory perceptual performance. J Neurosci 27:10734-10741.
Sur M, Garraghty PE, Roe AW (1988) Experimentally induced visual projections into auditory thalamus and cortex. Science 242:1437-1441.

Vaudano AE, Laufs H, Kiebel SJ, Carmichael DW, Hamandi K, Guye M, Thornton R, Rodionov R, Friston KJ, Duncan JS, Lemieux L (2009) Causal hierarchy within the thalamo-cortical network in spike and wave discharges. PLoS One 4:e6475.

von Melchner L, Pallas SL, Sur M (2000) Visual behaviour mediated by retinal projections directed to the auditory pathway. Nature 404:871876.

Weeks R, Horwitz B, Aziz-Sultan A, Tian B, Wessinger CM, Cohen LG, Hallett M, Rauschecker JP (2000) A positron emission tomographic study of auditory localization in the congenitally blind. J Neurosci 20:2664-2672.

Werner S, Noppeney U (2010) Distinct functional contributions of primary sensory and association areas to audiovisual integration in object categorization. J Neurosci 30:2662-2675.

Wittenberg GF, Werhahn KJ, Wassermann EM, Herscovitch P, Cohen LG (2004) Functional connectivity between somatosensory and visual cortex in early blind humans. Eur J Neurosci 20:1923-1927.

Yu C, Liu Y, Li J, Zhou Y, Wang K, Tian L, Qin W, Jiang T, Li K (2008) Altered functional connectivity of primary visual cortex in early blindness. Hum Brain Mapp 29:533-543. 